\title{
Efeito da reeducação postural global no alinhamento corporal e nas condições clínicas de indivíduos com disfunção temporomandibular associada a desvios posturais
}

\author{
Effect of global postural reeducation on body alignment and on clinical status of \\ individuals with temporomandibular disorder associated to postural deviations
}

Estudo desenvolvido no Laboratório de Motricidade Orofacial do Serviço de Atendimento Fonoaudiológico da UFSM - Universidade Federal de Santa Maria, Santa Maria, RS, Brasil

1 Fisioterapeuta Ms. da Prefeitura Municipal de Bagé, RS

2 Fisioterapeuta; Profa. Dra. adjunta do Depto. de Fisioterapia e Reabilitação da UFSM

3 Fonoaudióloga; Profa. Dra. adjunta do Depto. de Fonoaudiologia da UFSM

ENDEREÇO PARA CORRESPONDÊNCIA

Débora Basso

R. Gomes Carneiro 380 apt.402

Bairro Dores

97050-470 Santa Maria RS

e-mail:

deborababas@yahoo.com.br

APRESENTAÇÃO

ago. 2009

ACEITO PARA PUBLICAÇÃO

jan. 2010
Débora Basso, Eliane Corrêa ${ }^{2}$, Ana Maria da Silva ${ }^{3}$

Resumo: Este estudo visou verificar o efeito da técnica de reeducação postural global (RPG) nas condições físicas, psicológicas e psicossociais, assim como no alinhamento corporal, de indivíduos com disfunção temporomandibular (DTM) associada a desvio postural. Participaram 20 indivíduos com DTM e com desvio postural confirmado por exame físico, avaliados, antes e depois do tratamento de RPG, pelos critérios diagnósticos de desordens temporomandibulares (RDC/TMD, na sigla em inglês) e quanto às medidas angulares, por fotogrametria digital. $\mathrm{O}$ tratamento consistiu em 10 sessões semanais de RPG. Os resultados após o tratamento mostram, na classificação da disfunção, maior predomínio de desordens apenas musculares (em detrimento das articulares e por deslocamento de disco) e redução da intensidade da dor orofacial; o percentual de indivíduos sem depressão aumentou de $10 \%$ para $35 \%$; o percentual de indivíduos com classificação normal de sintomas físicos (excluindo itens de dor) passou de 30\% para 55\%. Foi encontrada melhora estatisticamente significante na maioria das medidas angulares, exceto nos ângulos frontais dos membros inferiores e ângulo perna/retropé direito. O alinhamento horizontal da cabeça e as medidas de lordose cervical e lombar, com valores normais antes da RPG, não se modificaram. Conclui-se que, com o tratamento de RPG, os indivíduos apresentaram importantes melhoras dos sintomas físicos e psicológicos da DTM, assim como melhora do alinhamento e simetria corporais.

Descritores: Modalidades de fisioterapia; Postura; Transtornos da articulação temporomandibular/reabilitação

ABstract: The purpose of this study was to assess the effects of the technique of global postural re-education (GPR) on body alignment and clinical status of individuals with temporomandibular disorder (TMD) associated to postural deviations. Twenty individuals with both TMD and postural deviations confirmed by physical examination were assessed, before and after treatment, by the research diagnostic criteria for temporomandibular disorders (RDC/TMD) and as to body angle measures, by digital photogrammetry. Treatment consisted of 10 weekly, 45minute GPR sessions. Results after treatment show higher predominance of myogenic dysfunction (rather than joint or disc ones) and significant orofacial pain intensity relief; the percentage of individuals without depression raised from $10 \%$ to $35 \%$; the percentage of individuals with physical symptoms classified as normal (excluding pain items) raised from 30 to $55 \%$. A statistically significant improvement was found in most body structures angle measures, except at lower limb frontal angles and at the right leg/foot angle. Head alignment and measures of cervical and lumbar lordosis, having normal values before treatment, did not change. Hence the GPR treatment may be said to have brought significant relief of TMD physical and psychological symptoms, as well as improvement in body alignment and symmetry. Key words: Physical therapy modalities; Posture; Temporomandibular joint disorders/rehabilitation 


\section{INTRODUÇÃO}

Disfunções temporomandibulares (DTM) são um grupo de condições dolorosas orofaciais que envolvem fatores de predisposição, início e perpetuação ${ }^{1,2}$. A associação entre desvios posturais dos ombros, coluna cervical, cabeça e outros segmentos corporais podem levar à disfunção craniocervical e, posteriormente, perpetuam os sinais e sintomas de $\mathrm{DTM}^{1,3}$. Os músculos mastigatórios têm íntima relação com a postura corporal, por meio de complexas conexões neuromusculares. Assim, alterações na articulação temporomandibular (ATM) podem influenciar o alinhamento postural ${ }^{4,5}$.

Devido à complexidade das DTM, faz-se necessário o diagnóstico correto e precoce, para prevenir danos às funções estomatognáticas ${ }^{6}$. Dentre os métodos de avaliação, destacam-se os critérios de diagnóstico em pesquisa para disfunções temporomandibulares (RDC) TMD, na sigla em inglês), que possibilitam múltiplos diagnósticos ou diagnósticos diferentes para cada ATM, bem como classificar subtipos de DTM ${ }^{6,7}$

Considera-se que uma criteriosa avaliação postural possa determinar os desvios e uma abordagem terapêutica dirigida para sua correção; vários estudos analisaram a relação entre postura corporal e DTM ${ }^{1,3,4,5,8-11}$. A combinação da fotografia digital a programas de informática permite medir ângulos e distâncias, como pelo software para avaliação postural SAPo ${ }^{12}$. Esse método de avaliação quantitativo possibilita identificar desvios posturais e suas possíveis complicações, permitindo ainda a comparação de valores obtidos após terapias $^{12,13}$.

Considerando que os desvios posturais desorganizam a harmonia corporal, com possíveis reflexos no sistema crânio-cérvico-mandibular 5,10,14, modalidades fisioterapêuticas como exercícios posturais, eletroterapia, reeducação proprioceptiva, entre outras, parecem beneficiar tanto as DTM quanto os desvios posturais ${ }^{14,15}$. Nessas modalidades inclui-se a reeducação postural global (RPG) ${ }^{16-18}$ que, com base na noção de integração das cadeias musculares, propõe uma atuação terapêutica de alongamentos visando o equilíbrio das tensões miofasciais e da postura corporal como um todo ${ }^{18-21}$. Apesar de ser amplamente utilizada na prática clínica, poucos estudos comprovam os resultados terapêuticos da RPG ${ }^{19,20,21}$. Algumas pesquisas ${ }^{10,14-16}$ restringem-se a orientações posturais para pacientes com DTM.

Assim, este estudo visou verificar o efeito da RPG no alinhamento corporal, bem como em condições físicas, psicológicas e em aspectos psicossociais de indivíduos com DTM associada a desvio postural.

\section{METODOLOGIA}

A coleta dos dados foi iniciada após aprovação do estudo pelo Comitê de Ética em Pesquisa da Universidade Federal de Santa Maria. Todos os participantes assinaram o termo de consentimento livre e esclarecido.

Com a divulgação da pesquisa em jornal local, no site da universidade e por contatos com o Serviço de Odontologia da universidade, 68 indivíduos apresentaram-se para participar. Foram considerados os seguintes critérios de inclusão: indivíduos de ambos os sexos, com idade entre 20 e 35 anos; DTM diagnosticada pelo RDC/TMD, associada a desvio postural, verificado por exame físico (protocolo de Kendall ${ }^{22}$ ). Foram excluídos indivíduos com comprometimento neuropsicomotor, doença musculoesquelética, traumas ortopédicos, cirurgia prévia ou má formação na região orofacial, e que estivessem ou tivessem estado nos últimos seis meses sob tratamento fisioterapêutico, fonoaudiológico e/ou odontológico. Com base nesses critérios, 20 indivíduos foram selecionados para participar do estudo.

Para o diagnóstico da DTM utilizou-se o questionário RDC/TMD. Desenvolvido por Dworkin e Le Resche ${ }^{6}$, contempla no eixo I os aspectos físicos, classificandoos em: grupo I, desordem muscular; grupo II, deslocamento de disco; e grupo III, outras condições articulares. O eixo II contempla aspectos psicossociais: gradua a intensidade de dor orofacial crônica em grau 0, sem dor de DTM nos seis meses prévios; grau I, baixa incapacidade e intensidade; grau II, baixa incapacidade e alta intensidade; grau III, alta incapacidade e limitação moderada; e grau IV, alta incapacidade e limitação severa. O eixo II permite ainda classificar como normal, moderados ou severos sintomas de depressão e sintomas físicos não-específicos.

A avaliação postural foi feita por fotografias com câmera digital (Sony DSC-S40, resolução de 4.1 megapixels), posicionada paralelamente a um metro do chão, sobre um tripé (Vanguard). Os sujeitos foram posicionados a três metros da câmera fotográfica nas vistas anterior, posterior e perfil esquerdo. Permaneceram na postura habitualmente adotada e, para calibrar a fotografia no programa e como referência para o alinhamento corporal, utilizou-se um fio de prumo fixado ao teto da sala.

As referências anatômicas foram manualmente palpadas e demarcadas com bolas de isopor e fita adesiva duplaface, de acordo com o software para avaliação postural SAPo $(v 0.68)^{12}$, bilateralmente, na vista anterior em: tragus, acrômio, espinha ilíaca ântero-superior (EIAS), trocânter maior, projeção lateral da linha articular do joelho, centro da patela, tuberosidade da tíbia, maléolos laterais, maléolos mediais; na vista posterior em: ângulo inferior da escápula, terceira vértebra torácica (T3), ponto medial da perna, linha intermaleolar e tendão do calcâneo. Na vista lateral esquerda, foram marcados: tragus, sétima vértebra cervical (C7), acrômio, EIAS, espinha ilíaca póstero-superior, trocânter maior, projeção lateral da linha articular do joelho, maléolo lateral, região entre o segundo e o terceiro metatarso.

A avaliação das curvas da coluna cervical e lombar também foi feita por fotografia digital, com um traçado a partir do plano vertical que tangencia a região mais proeminente da cifose torácica ${ }^{1,4}$.

\section{Reeducação postural global}

Os participantes submeteram-se a 10 sessões de RPG com duração de 45 minutos, uma vez por semana, adotandose duas posturas por sessão de terapia ${ }^{18}$. Inicialmente, foram trabalhadas posturas sem carga. Após os pacientes apresentarem redução das compensações, resistências e alcançarem melhor alinhamento dos segmentos corporais, foram realizadas 
posturas com carga ${ }^{18,20}$. Nas sessões, foi enfatizada a postura mais indicada para cada paciente, bem como a função da cadeia inspiratória ${ }^{18,19,21}$. Utilizou-se como critério na escolha das posturas de cada participante, em cada sessão, a postura global na qual a correção fosse mais difícil de ser mantida ${ }^{18}$.

As avaliações e o tratamento foram efetuados sempre pela mesma fisioterapeuta. Ao final, foram realizadas reavaliações pelo RDC/TMD e por biofotogrametria.

\section{Análise dos dados}

A análise qualitativa dos resultados do RDC/TMD foi feita por distribuição da freqüência das alterações. As fotografias digitais foram analisadas por meio do SAPo, que fornece automaticamente o cálculo das medidas angulares. Os valores de referência do software são: zero grau para alinhamento vertical e horizontal da cabeça, dos acrômios, das EIAS, das tuberosidades das tíbias, ângulo entre os acrômios e EIAS, assimetria das escápulas em relação à T3, e plano frontal e sagital; 15 graus para os ângulos $\mathrm{Q}$ direito e esquerdo; ausência de diferença de comprimento dos membros inferiores $(0,0 \mathrm{~cm})^{12}$.

Para o ângulo frontal do membro inferior e ângulo entre a perna e o retropé, o alinhamento vertical do tronco e do corpo, o alinhamento horizontal da pélvis, ângulos do quadril, do joelho e do tornozelo, sem referências estabelecidas pelo programa, compararam-se os valores antes e após a RPG utilizando-se o alinhamento em relação ao fio de prumo. Assim, nas vistas anterior e posterior, o fio de prumo deveria passar por entre os maléolos mediais, estender-se entre os membros inferiores (MMII) passando pela linha média da pelve, coluna e cabeça. Na vista lateral, este deveria passar ligeiramente anterior ao maléolo lateral e à articulação do joelho; levemente posterior à articulação do quadril, próximo ao meio do tronco, no meio do ombro, e pelo conduto auditivo externo ${ }^{22}$.

O alinhamento horizontal da cabeça, em perfil, foi avaliado pelo ângulo formado pela C7, linha horizontal e tragus, conforme o software SAPo. Como valor de referência, uma vez que não se en- contra descrito no software, foi considerado o ângulo de $48,9 \pm 6,5^{\circ}$, conforme estudo de Raine e Twomey ${ }^{23}$.

Como parâmetro de normalidade para a curva cervical, considerou-se a distância do plano da cifose torácica de 6 a $8 \mathrm{~cm}$ e, para a curva lombar, de 4 a 6 $\mathrm{cm}$. Valores abaixo destes para cada região foram consideradas curvas diminuídas, sendo valores acima destes curvas lordóticas aumentadas ${ }^{1,4}$.

Analisaram-se os dados fotogramétricos por meio do teste de Lilliefors. Como tais dados não seguiram distribuiçãa normal, efetuou-se análise pelo teste nãoparamétrico de Wilcoxon. Foi considerada significância de 5\%.

\section{RESULTADOS}

Pelos resultados do eixo I do RDC/ TMD, 100\% dos participantes apresentavam DTM; na avaliação inicial, 55\%

Tabela 1 Distribuição (\%) do diagnóstico de DTM nos diferentes subgrupos do eixo I do RDC/TMD, antes (pré) e depois (pós) do programa de RPG - reeducação postural global $(n=20)$

\begin{tabular}{lcc}
\hline Diagnóstico & Pré-RPG $(\%)$ & Pós-RPG $(\%)$ \\
\hline Grupo I & 55 & 75 \\
Grupos I e II & 5 & 5 \\
Grupos I e III & 35 & 15 \\
Grupos I, II e III & 5 & 0 \\
Sem diagnóstico & 0 & 5 \\
\hline
\end{tabular}

RDC/TMD = critérios de diagnóstico em pesquisa para disfunções

temporomandibulares; Grupo I = desordem muscular; Grupo II = deslocamento de disco; Grupo III = outras condições articulares deles foram classificados no grupo I. Ao final do tratamento, em um participante observou-se completa extinção da disfunção; e aumentou bastante o predomínio da classificação no grupo I (75\%, Tabela 1).

No eixo II, verificou-se redução da intensidade da dor orofacial crônica. Observou-se melhora dos sintomas físicos incluindo itens de dor, sendo que a classificação "normal" passou de 10 para $30 \%$ dos pacientes. Nos sintomas físicos excluindo itens de dor, a classificação "normal" passou a abranger 55\%; o percentual de indivíduos sem depressão aumentou de 10 para 35\% após a RPG (Tabela 2).

Quanto à postura corporal, no exame físico realizado pelo protocolo de Kendall22, observaram-se os seguintes desvios: anteriorização e inclinação lateral da cabeça, rotação e elevação dos ombros, retificação da coluna cervical, flexão de cotovelos, hiperlordose lombar, rotação de pélvis, hiperextensão de joelhos e pés pronados. Após a RPG, encontraram-se diferenças significantes, para melhor, em 19 das 25 medidas angulares calculadas. Na vista anterior, houve melhora do alinhamento horizontal da cabeça, dos acrômios, das EIAS, e das tuberosidades das tíbias; dos ângulos entre os acrômios e EIAS, Q direito e esquerdo; da diferença de comprimento dos MMII, e da assimetria do plano frontal (Tabela 3). $\mathrm{Na}$ vista posterior, houve melhora da assimetria horizontal das escápulas em relação à T3 e no ângulo entre a perna e o retropé esquerdo (Tabela 4). Na vista lateral esquerda, verificou-se melhora significante do alinhamento vertical da cabeça, do tronco e do corpo; do alinhamento horizontal da pélvis; dos ângulos do quadril, do joelho e do tornozelo, e na assimetria do plano sagital (Tabela 5).

Tabela 2 Distribuição (\%) da classificação dos sintomas diagnosticados pelo eixo II do RDC/TMD, antes (pré) e depois (pós) do programa de RPG reeducação postural global $(n=20)$

\begin{tabular}{lcccccc}
\hline $\begin{array}{l}\text { Classifi- } \\
\text { cação }\end{array}$ & \multicolumn{2}{c}{ SFID } & \multicolumn{2}{c}{ SFED } & \multicolumn{2}{c}{ Depressão } \\
\hline Pormal & 10 & 30 & 30 & 55 & 10 & 35 \\
Moderado & 40 & 50 & 35 & 40 & 50 & 40 \\
Severo & 50 & 20 & 35 & 5 & 40 & 25 \\
\hline
\end{tabular}

RDC/TMD = critérios de diagnóstico em pesquisa para disfunções temporomandibulares; SFID = sintomas físicos incluindo itens de dor; SFED = sintomas físicos excluindo itens de dor 
Tabela 3 Medidas da avaliação postural (média \pm desvio padrão) na vista anterior, antes (pré) e depois (pós) do programa de reeducação postural global (RPG), e valor de $p$ da comparação entre pré e pós $(n=20)$

\begin{tabular}{lcrc}
\hline Item postural da vista anterior & Pré-RPG & Pós-RPG & $p$ \\
\hline Alinh horiz cabeça $\left(^{\circ}\right)$ & $1,72 \pm 1,71$ & $0,59 \pm 0,90$ & 0,0022 \\
Alinh horiz acrômios $\left(^{\circ}\right)$ & $1,35 \pm 1,08$ & $0,68 \pm 0,57$ & 0,0014 \\
Alinh horiz EIAS $\left(^{\circ}\right)$ & $1,35 \pm 0,70$ & $0,64 \pm 0,71$ & 0,0006 \\
Ângulo acrômios/EIAS $\left(^{\circ}\right)$ & $2,15 \pm 1,46$ & $0,99 \pm 0,84$ & 0,0004 \\
Ângulo frontal do MID $\left(^{\circ}\right)$ & $2,43 \pm 1,76$ & $2,35 \pm 1,39$ & 0,9030 \\
Ângulo frontal do MIE $\left(^{\circ}\right)$ & $3,20 \pm 2,46$ & $3,21 \pm 2,67$ & 0,4781 \\
Diferença comprimento MMII $(\mathrm{cm})$ & $1,04 \pm 0,50$ & $0,52 \pm 0,40$ & 0,0002 \\
Alinh horiz tuberosidades da tíbia $\left(^{\circ}\right)$ & $2,08 \pm 1,48$ & $0,89 \pm 0,97$ & 0,0006 \\
Ângulo Q direito $\left({ }^{\circ}\right)$ & $21,17 \pm 8,24$ & $18,85 \pm 5,94$ & 0,0130 \\
Ângulo Q esquerdo $\left({ }^{\circ}\right)$ & $25,24 \pm 7,67$ & $22,90 \pm 7,40$ & 0,0008 \\
Assimetria no plano frontal $(\%)$ & $0,10 \pm 0,13$ & $0,06 \pm 0,07$ & 0,0333
\end{tabular}

Alinh horiz = alinhamento horizontal; EIAS = espinha ilíaca ântero-superior; MID = membro inferior direito; $\mathrm{MIE}=$ membro inferior esquerdo; $\mathrm{MMII}=$ membros inferiores

Tabela 4 Medidas da avaliação postural (média \pm desvio padrão) na vista posterior, antes (pré) e depois (pós) do programa de reeducação postural global (RPG), e valor de $\mathrm{p}$ da comparação entre pré e pós $(\mathrm{n}=20)$

\begin{tabular}{lccc}
\hline Item postural da vista posterior & Pré-RPG & Pós-RPG & $p$ \\
\hline Assimetria horiz escápulas/T3 $(\%)$ & $18,55 \pm 12,71$ & $7,08 \pm 6,99$ & 0,0000 \\
Ângulo perna/retropé D $\left(^{\circ}\right)$ & $20,80 \pm 8.31$ & $17,69 \pm 7,04$ & 0,1789 \\
Ângulo perna/retropé E $\left(^{\circ}\right)$ & $22,06 \pm 7,40$ & $16,08 \pm 7,34$ & 0,0186 \\
\hline
\end{tabular}

Horiz = horizontal; $\mathrm{T} 3$ = terceira vértebra torácica; $\mathrm{D}=$ direito; $\mathrm{E}=$ esquerdo

Tabela 5 Medidas da avaliação postural (média \pm desvio padrão) na vista lateral esquerda, antes (pré) e depois (pós) do programa de reeducação postural global (RPG), e valor de p da comparação entre pré e pós $(n=20)$

\begin{tabular}{lccc}
\hline Item postural da vista lateral esquerda & Pré-RPG & Pós-RPG & $p$ \\
\hline Alinh horiz cabeça em relação à C7 $\left(^{\circ}\right)$ & $52,46 \pm 5,86$ & $54,15 \pm 3,85$ & 0,1262 \\
Alinh vertical cabeça em relação aos & $11,14 \pm 7,61$ & $9,00 \pm 5,66$ & 0,0001 \\
acrômios $\left(^{\circ}\right)$ & $2,52 \pm 2,81$ & $1,31 \pm 1,12$ & 0,0004 \\
Alinh vertical do tronco $\left(^{\circ}\right)$ & $6,78 \pm 3,78$ & $7,74 \pm 3,00$ & 0,0382 \\
Ângulo do quadril $\left(^{\circ}\right)$ & $1,53 \pm 0,79$ & $0,91 \pm 0,56$ & 0,0004 \\
Alinh vertical do corpo $\left(^{\circ}\right)$ & $9,13 \pm 8,43$ & $8,24 \pm 9,69$ & 0,0017 \\
Alinh horiz pélvis $\left(^{\circ}\right)$ & $4,89 \pm 4,11$ & $2,52 \pm 2,85$ & 0,0001 \\
Ângulo do joelho $\left(^{\circ}\right)$ & $88,53 \pm 2,52$ & $86,06 \pm 2,94$ & 0,0001 \\
Ângulo do tornozelo $\left(^{\circ}\right)$ & $0,26 \pm 0,07$ & $0,20 \pm 0,08$ & 0,0000 \\
Assimetria no plano sagital $(\%)$ & $6,15 \pm 1,50$ & $6,64 \pm 0,83$ & 0,0853 \\
Lordose cervical $(\mathrm{cm})$ & $4,13 \pm 1,23$ & $4,61 \pm 0,57$ & 0,1031 \\
Lordose lombar $(\mathrm{cm})$ & A &
\end{tabular}

Alinh $=$ alinhamento; Horiz $=$ horizontal; $\mathrm{C} 7$ = sétima vértebra cervical

\section{DISCUSSÃO}

Várias pesquisas mostram associação entre desvios posturais e DTM ${ }^{1,3,5,8,9}$. Neste estudo, o exame físico permitiu constatar anteriorização e inclinação lateral da cabeça, rotação e elevação dos ombros, retificação da coluna cervical, flexão de cotovelos, hiperlordose lombar, rotação de pélvis, hiperextensão de joelhos e pés pronados, como os principais desvios posturais apresentados.

Os resultados deste estudo mostraram predomínio da desordem muscular antes (55\%) e após (75\%) a RPG (eixo I do RDC/TMD). O maior número de pacien- tes com DTM miogênica após o tratamento indica redução dos sinais e/ou sintomas de deslocamento de disco e das desordens articulares, pois se observou modificação das disfunções mistas para apenas musculares. Também ocorreu extinção da disfunção em um participante ao final do tratamento. Atribui-se isso ao fato de que o método de RPG aborda a postura global, atuando nos músculos, ligamentos e articulações de forma nãolocalizada, ou seja, sem enfatizar a musculatura mastigatória e cervical.

Também se verificou que a intensidade da dor crônica e a incapacidade foram minoradas, em consonância com a pesquisa de Maluf ${ }^{19}$, que comparou o efeito da RPG e do alongamento estático segmentar em portadoras de DTM. A autora verificou que as técnicas foram igualmente eficazes na redução dos sintomas e no aumento do limiar de dor dos músculos mastigatórios e cervicais.

Os sintomas físicos não-específicos e a depressão tiveram importante melhora após a RPG. Examinado a relação entre DTM e depressão, Toledo et al. ${ }^{24}$ verificaram depressão moderada ou grave nos pacientes com DTM grave e, nos participantes com depressão grave, algum tipo de DTM. Os autores concluem que depressão pode ser fator etiológico de DTM, pois houve associação significante entre esta e o grau de depressão.

Após o tratamento, os sinais e sintomas de DTM se atenuaram com tendência à normalidade, nos grupos de DTM mista. Tal resultado pode dever-se à melhora do diagnóstico de artralgia, apesar da disfunção miogênica ainda estar presente. Resultados semelhantes foram encontrados em estudos que utilizaram terapia manual, eletroterapia e correção postural para a redução ou cessação de dor na ATM e coluna cervical, para a meIhora da amplitude de abertura da boca e da função mandibular, e correção postural $^{10,14,15}$.

Nesta pesquisa, pela biofotogrametria, observou-se melhora do alinhamento horizontal (Tabela 3) e vertical da cabeça (Tabela 5), o que pode ter sido responsável pela redução dos sintomas detectada pelo RDC/TMD. A relação entre os desvios posturais da cabeça e a DTM também é referida em outros estudos ${ }^{1,3,5,8}$. Porém, lunes et al. ${ }^{11}$, em es- 
tudo com fotogrametria, radiografias e observação visual, não encontraram diferenças posturais da cabeça e coluna cervical em indivíduos com e sem DTM.

Observou-se, na vista anterior, no alinhamento horizontal da cabeça (correção de rotações), dos acrômios, das EIAS e das tuberosidades das tíbias, maior simetria dos pontos anatômicos em relação à referência do SAPo. Esse resultado demonstra que desvios posturais geram tensão nas cadeias musculares e, por seqüência, nos músculos mastigatórios e cervicais, podendo influenciar a DTM. Resultados semelhantes foram encontrados por Yi et al. ${ }^{1}$, que observaram, por meio de imagens fotográficas, a relação entre a hiperatividade desses músculos e a postura corporal. A melhora significativa na medida do ângulo entre acrômios e EIAS, em relação ao padrão de referência $\left(0^{\circ}\right)$, demonstra redução da inclinação do tronco; e, na diferença de comprimento dos MMII, bem como no ângulo $\mathrm{Q}$ direito e esquerdo, indica correção de alinhamento pélvico e do valgismo de joelho, uma vez que o aumento desse ângulo está associado a esse desvio ${ }^{13}$.

Os resultados acima mostram que a correção dos desvios relaciona-se com a adequação da postura da cabeça, indicando benefícios na simetria corporal de forma global. Isso está de acordo com os achados de Tecco et al. ${ }^{25}$, que estudaram o efeito da lesão do ligamento cruzado anterior na atividade muscular dos músculos da cabeça, pescoço e tronco. Os autores referem que desvios nas extremidades inferiores podem resultar em alterações neuromusculares e articulares superiores, enfatizando a complexa relação entre os músculos da cabeça, pescoço e tronco e as lesões de joelho.
A redução da medida de assimetria do plano frontal e sagital sugere um meIhor equilíbrio na distribuição das pressões plantares de contato. Isso concorda com o estudo de Teodori et al..$^{21}$, sobre a eficácia da RPG na recuperação da simetria corporal, para readequação das pressões de contato e sua interferência positiva na distribuição do centro de força. A diferença significante observada apenas no ângulo entre a perna e o retropé esquerdo pode ser explicada pelo fato de o calcâneo esquerdo ter apresentado maior valgismo antes da RPG.

A redução do índice de assimetria entre as escápulas e T3, na vista posterior, com tendência à referência $(0 \%)$, indica melhor simetria escapular. Pita ${ }^{17}$ descreve o caso de um paciente com cifose torácica, lombalgia crônica e outros desvios posturais, submetido à RPG, que apresentou melhora dos desvios, inclusive no alinhamento escapular.

$\mathrm{O}$ alinhamento horizontal da cabeça em relação à $\mathrm{C} 7$, quando reduzido, determina a posição anteriorizada da cabeça. Raine e Twomey ${ }^{23}$ verificaram valores de $48,9^{\circ}\left( \pm 6,5^{\circ}\right)$ em indivíduos assintomáticos. Os resultados deste estudo concordam com esses autores, pois os valores angulares encontrados foram similares a estes, antes e após RPG. Isso justifica o fato de que não houve diferença significante após a RPG. Porém, cabe ressaltar que no exame físico a anteriorização da cabeça foi evidenciada.

Nos demais alinhamentos e ângulos obteve-se melhora significativa após a RPG. Isso sugere uma repercussão positiva do método nos desvios posturais de portadores de DTM. Ainda, por conseqüência, pode ter contribuído na redu- ção dos sinais e sintomas observados no eixo II do RDC/TMD. Tais achados estão de acordo com Maluf ${ }^{19}$, que verificou resultados positivos nos sintomas da DTM devido à atuação global da RPG.

As medidas no plano torácico, da lordose cervical e lordose lombar, não se modificaram, pois já estavam dentro da normalidade. Esses resultados contrariam o estudo de Yi et al. ${ }^{1}$, que encontraram associação entre a hiperlordose cervical e tensão da musculatura mastigatória em indivíduos com DTM; no entanto, corroboram sua análise da lordose lombar, em que não encontraram relação com a DTM. Porém, lunes et al. ${ }^{11}$, em uma análise visual da postura craniocervical, observaram retificação da coluna cervical, tanto em indivíduos com essa disfunção quanto assintomáticos.

\section{CONCLUSÃO}

Os portadores de DTM associada a desvios posturais se beneficiaram do tratamento proposto pelo método de RPG: boa parte dos que apresentavam desordens articulares e do disco articular passaram a apresentar apenas disfunção muscular; houve redução da incapacidade e intensidade da dor crônica, melhora dos sintomas físicos não-específicos e da depressão, com tendência à normalidade. As medidas do alinhamento horizontal da cabeça em relação à $\mathrm{C} 7$ e a lordose cervical e lombar pouco se modificaram, pois os valores já eram fisiológicos antes da RPG; no entanto, houve melhora significante no alinhamento das escápulas, acrômios e EIAS, na simetria e ângulos dos MMII e na posição da cabeça. 


\section{REFERENCIAS}

1 Yi LC, Guedes ZCF, Vieira MM. Relação da postura corporal com a disfunção da articulação temporomandibular: hiperatividade dos músculos da mastigação. Rev Fisioter Bras. 2003;4:341-7.

2 Matta MAP, Honorato DC. Uma abordagem fisioterapêutica nas desordens temporomandibulares: estudo retrospectivo. Rev Fisioter Univ São Paulo. 2003;10:77-83.

3 Bevilaqua-Grossi D, Chaves TC, Oliveira AS. Cervical spine signs and symptoms: perpetuating rather than predisposing factors for temporomandibular disorders in women. J Appl Oral Sci. 2007;15:259-64.

4 Bricot, B. Postura normal e postura patológica: posturologia. São Paulo: Ícone; 2001.

5 Amantéa DV, Novaes AP, Campolongo GD, Barros TP. A importância da avaliação postural no paciente com disfunção da articulação temporomandibular. Acta Ortop Bras. 2004;12:155-9.

6 Dworkin SF, Le Resche L. Research diagnostic criteria for temporomandibular disorders: review, criteria, examinations and specifications, critique. J Craniomandib Disord. 1992;6:301-55.

7 Lucena LBS, Kosminsky M, Costa LJ, Góes PSA. Validation of the portuguese version of the RDC/TMD axis II questionnaire. Braz Oral Res. 2006;20:312-7.

8 Munhoz WC, Marques AP, Siqueira JTT. Evaluation of body posture in individuals with internal temporomandibular joint derangement. Cranio. 2005;23:269-77.

9 Munhoz WC, Marques AP. Body posture evaluations in subjects with internal temporomandibular joint derangement. Cranio. 2009;27(4):231-42.

10 Olivo AS, Bravo J, Magee DJ, Thie NMR, Major PW, Flores-Mir C. The association between head and cervical posture and temporomandibular disorders: a systematic review. J Orofac Pain. 2006;20:9-23.

11 Iunes DH, Carvalho LCF, Oliveira AS, Bevilaqua-Grossi D. Análise da postura craniocervical em pacientes com disfunção temporomandibular. Rev Bras Fisioter. 2009;13(1):89-95.

12 SAPO v.0.68: Portal do projeto Software para Avaliação Postural [homepage na Internet]. SãoPaulo: Incubadora Virtual Fapesp; 2007 [citado set 2007]. Disponível em: http://sapo.incubadora.fapesp.br/portal.
13 Sacco ICN, Alibert S, Queiroz BWC, Pripas D, Kieling I, Kimura AA, et al. Confiabilidade da fotogrametria em relação à goniometria para avaliação postural de membros inferiores. Rev Bras Fisioter. 2007;11:411-7.

14 Nicolakis P, Erdogmus B, Kopf A, Djaber-Ansari A, Piehslinger E, Fialka-Moser V. Exercise therapy for cranomandibular disorders. Arch Phys Med Rehabil. 2000;81:1137-42.

15 Medlicott MS, Harris SR. A systematic review of the effectiveness of exercise, manual therapy, electrotherapy, relaxation training, and biofeedback in the management of temporomandibular disorder. Phys Ther. 2006;86:955-73.

16 Marques AP. Escoliose tratada com reeducação postural global. Rev Fisioter Univ São Paulo. 1996;3:65-8.

17 Pita MC. Cifose torácica tratada com reeducação postural global. Arq Cienc Saude Unipar. 2000;4:159-63.

18 Souchard P E. Fundamentos da reeducação postural global: princípios e originalidade. São Paulo: É Realizações; 2003.

19 Maluf SA. Efeito da reeducação postural global e do alongamento estático em portadoras de disfunção temporomandibular: um estudo comparativo [tese]. São Paulo: Faculdade de Medicina, Universidade de São Paulo; 2006.

20 Vanti C, Generali A, Ferrari S, Nava T, Tosarelli D, Pillastrini P. La rieducazione posturale globale nelle patologie musculo-scheletriche: evidenze scientifiche e indicazione cliniche. Rev Reumatol. 2007;59:192-201.

21 Teodori RM, Guirro ECO, Santos RM. Distribuição da pressão plantar e localização do centro de força após intervenção pelo método de reeducação postural global: um estudo de caso. Fisioter Mov. 2005; 18:27-35.

22 Kendall FP, McCreary EK, Provance PG, Rodgers MM, Romani WA. Músculos: provas e funções; com postura e dor. 5a ed. São Paulo: Manole; 2007.

23 Raine S, Twomey LT. Head and shoulder posture variations in 160 asymptomatic women and men. Arch Phys Med Rehabil. 1997;78:1215-23.

24 Toledo BAS, Capote TSO, Campos JADB. Associação entre disfunção temporomandibular e depressão. Cienc Odontol Bras. 2008;11:75-9.

25 Tecco S, Vincenzo S, Stefano T, Felice F. Effects of anterior cruciate ligament $(\mathrm{ACL})$ injury on muscle activity of head, neck and trunk muscles: a crosssectional evaluation. Cranio. 2007;25(3):177-85. 